\title{
Correction to: Single Plasmid-Based, Upgradable, and Backward-Compatible Adenoviral Vector Systems, by Liu H, Lu Z, Zhang X, Guo X, Mei L, Zou X, Zhong Y, Wang $M$, and Hung $T$. Hum Gene Ther 2019:30(6):777-791.DOI: 10.1089/hum.2018.258
}

IN THE JUNE 2019 ISSUE of Human Gene Therapy (vol. 30, no. 6, pp. 777-791), the article "Single PlasmidBased, Upgradable, and Backward-Compatible Adenoviral Vector Systems," by Hongyan Liu et al., contains an error in the correspondence information. Dr. Zhuozhuang Lu should have been listed as the primary contact, while Dr. Yuxu Zhong should have been secondary.

The original correspondence information read:

\begin{abstract}
“*Correspondence: Dr. Yuxu Zhong, State Key Laboratory of Toxicology and Medical Counter Measures, Beijing Institute of Pharmacology and Toxicology, Beijing 100850, P.R. China. E-mail: yuxuzhong2008@aliyun.com; or Dr. Zhuozhuang Lu, State Key Laboratory of Infectious Disease Prevention and Control, National Institute for Viral Disease Control and Prevention, Chinese Center for Disease Control and Prevention, Beijing 100052, P.R. China. E-mail: luzz@ivdc.chinacdc.cn”
\end{abstract}

This should have appeared as:

*Correspondence: Dr. Zhuozhuang Lu, State Key Laboratory of Infectious Disease Prevention and Control, National Institute for Viral Disease Control and Prevention, Chinese Center for Disease Control and Prevention, Beijing 100052, P.R. China. E-mail: luzz@ivdc.chinacdc.cn; or Dr. Yuxu Zhong, State Key Laboratory of Toxicology and Medical Counter Measures, Beijing Institute of Pharmacology and Toxicology, Beijing 100850, P.R. China. E-mail: yuxuzhong 2008@aliyun.com

The online version of the article has been corrected to reflect these changes. 